\title{
Anderson Transition in Low-Dimensional Disordered Systems Driven by Long-Range Non random Hopping
}

\author{
A. Rodríguez, ${ }^{1}$ V. A. Malyshev, ${ }^{2, *}$ G. Sierra, ${ }^{3}$ M. A. Martín-Delgado, ${ }^{4}$ \\ J. Rodríguez-Laguna, ${ }^{4}$ and F. Domínguez-Adame ${ }^{2}$ \\ ${ }^{1}$ Departamento de Matemática Aplicada y Estadística, Universidad Politécnica, E-28040 Madrid, Spain \\ ${ }^{2}$ GISC, Departamento de Física de Materiales, Universidad Complutense, E-28040 Madrid, Spain \\ ${ }^{3}$ Instituto de Matemáticas y Física Fundamental, CSIC, Madrid, Spain \\ ${ }^{4}$ Departamento de Física Teórica I, Universidad Complutense, E-28040 Madrid, Spain
}

(Received 23 April 2002; published 17 January 2003)

\begin{abstract}
The single-parameter scaling hypothesis predicts the absence of delocalized states for noninteracting quasiparticles in low-dimensional disordered systems. We show analytically, using a supersymmetric method combined with a renormalization group analysis, as well as numerically that extended states may occur in the one- and two-dimensional Anderson model with a nonrandom hopping falling off as some power of the distance between sites. The different size scaling of the bare level spacing and the renormalized magnitude of the disorder seen by the quasiparticles finally results in the delocalization of states at one of the band edges of the quasiparticle energy spectrum.
\end{abstract}

DOI: $10.1103 /$ PhysRevLett.90.027404

Localization of noninteracting quasiparticles in random media with time-reversal symmetry and finiterange hopping have been extensively studied since the seminal paper by Anderson [1]. The hypothesis of singleparameter scaling, introduced in Ref. [2], led to the general belief that all eigenstates of noninteracting quasiparticles were exponentially localized in one (1D) and two (2D) dimensions (see Refs. [3,4] for a comprehensive review) and that localization-delocalization transitions no longer exist in the thermodynamic limit. Even though models with finite-range hopping work nicely in describing a variety of materials, long-range hopping is often found in different physical systems (e.g., Frenkel excitons). Random long-range hopping was found to give rise to delocalization of states not only in threedimensional systems [1] but also in any dimension [58]. Recent studies [9] revised the validity of the singleparameter scaling hypothesis even within the original 1D Anderson model with nearest-neighbor coupling, although did not question the statement that all eigenstates in 1D random systems are localized.

In this Letter, we present analytical and numerical proofs that a localization-delocalization transition may occur in 1D and 2D systems with diagonal disorder and nonrandom intersite coupling which falls off according to a powerlike law. Apart from the importance of this finding from a general point of view, it may be relevant for several physical systems. As an example, let us mention dipolar Frenkel excitons on 2D regular lattices where molecules are subjected to randomness due to a disordered environment [10]. Biological light-harvesting antenna systems represent a realization of the model we are dealing with [11,12]. Magnons in 1D and 2D disordered spin systems provide one more example of interest.
PACS numbers: 78.30.Ly, 36.20.Kd, 71.30.+h, 71.35.Aa
We consider the Anderson Hamiltonian on a $d$-dimensional $(d=1,2)$ simple lattice with $\mathcal{N}=N^{d}$ sites:

$$
\mathcal{H}=\sum_{\mathbf{n}} \varepsilon_{\mathbf{n}}|\mathbf{n}\rangle\left\langle\mathbf{n}\left|+\sum_{\mathbf{n m}} J_{\mathbf{n m}}\right| \mathbf{n}\right\rangle\langle\mathbf{m}|,
$$

where $|\mathbf{n}\rangle$ is the ket-vector of the state localized at site $\mathbf{n}$, and $\left\{\varepsilon_{\mathbf{n}}\right\}$ are random site energies, assumed to be uncorrelated for different sites and distributed uniformly within an interval $[-\Delta / 2, \Delta / 2]$, thus having zero mean and standard deviation $\sigma=\Delta / \sqrt{12}$. The hopping integrals between lattice sites $\mathbf{m}$ and $\mathbf{n}$ will be taken in the form $J_{\mathbf{m n}}=J /|\mathbf{m}-\mathbf{n}|^{\mu}\left(J_{\mathbf{m m}} \equiv 0\right)$, where $J>0$ without loss of generality and the lattice constant is set to unity. We stress that hopping integrals do not fluctuate.

For our qualitative reasoning we rewrite the Hamiltonian (1) in the Bloch wave representation, $|\mathbf{k}\rangle=$ $\mathcal{N}^{-1 / 2} \sum_{\mathbf{n}} \exp (i \mathbf{k n})|\mathbf{n}\rangle$, with periodic boundary conditions. It then reads

$$
\begin{aligned}
\mathcal{H} & =\sum_{\mathbf{k}} E_{\mathbf{k}}|\mathbf{k}\rangle\left\langle\mathbf{k}\left|+\sum_{\mathbf{k} \mathbf{k}^{\prime}}(\delta \mathcal{H})_{\mathbf{k} \mathbf{k}^{\prime}}\right| \mathbf{k}\right\rangle\left\langle\mathbf{k}^{\prime}\right|, \\
E_{\mathbf{k}} & =J \sum_{\mathbf{n} \neq \mathbf{0}} \frac{e^{i \mathbf{k n}}}{|\mathbf{n}|^{\mu}} \\
(\delta \mathcal{H})_{\mathbf{k} \mathbf{k}^{\prime}} & =\frac{1}{\mathcal{N}} \sum_{\mathbf{n}} \varepsilon_{\mathbf{n}} e^{i\left(\mathbf{k}-\mathbf{k}^{\prime}\right) \mathbf{n}},
\end{aligned}
$$

where the wave numbers $\mathbf{k}$ and $\mathbf{k}^{\prime}$ run over the first Brillouin zone. Notice that $\mu>d$ to ensure the convergence of (2b) in the thermodynamic limit.

The key point of our qualitative arguments is as follows [13]: We compare the size scaling of the typical magnitude of the scattering matrix $(\delta \mathcal{H})_{\mathbf{k k}^{\prime}}$ with the size scaling of the level spacing $\delta E$ in the bare quasiparticle 
spectrum of the homogeneous Hamiltonian $(\Delta=0)$. In particular, we focus our attention on those eigenstates lying close to the band edges $\mathbf{k}=0$ (top) and $\mathbf{k}=\boldsymbol{\pi}$ (bottom), where $\pi=\pi$ and $(\pi, \pi)$ for 1D and 2D systems, respectively. The typical fluctuation of the scattering matrix $(\delta \mathcal{H})_{\mathbf{k k}^{\prime}}$ is $\sigma_{\text {eff }}=\sigma / N^{d / 2}$. Thus, in spite of the fact that the magnitude of the disorder is $\sigma$, the quasiparticle sees an effectively reduced value $\sigma_{\text {eff }}$. It is important that $\sigma_{\text {eff }}$ scales inversely proportional to $N^{d / 2}$. Straightforward calculations of the bare energy spectrum (2b) close to the band edges give the following results:

$$
\begin{array}{ll}
E_{\mathbf{k}} \simeq E_{0}-J A_{d}(\mu)|\mathbf{k}|^{\mu-d}, & |\mathbf{k}| \rightarrow 0, \\
E_{\mathbf{k}} \simeq E_{\boldsymbol{\pi}}+J B_{d}(\mu)|\mathbf{k}-\boldsymbol{\pi}|^{2}, & \mathbf{k} \rightarrow \boldsymbol{\pi},
\end{array}
$$

for $\mu \neq d+2$, where $E_{0}$ and $E_{\pi}$ are the band-edge energies and $A_{d}(\mu)$ and $B_{d}(\mu)$ are dimensionless constants. From (3) it follows that the level spacing scales as $\delta E \sim$ $N^{-\mu+d}$ at the top of the band, while at the bottom one gets $\delta E \sim N^{-2}$.

The matrix $(\delta \mathcal{H})_{\mathbf{k k}^{\prime}}$ couples the bare (extended) quasiparticle states to each other and may result in their localization within a region of size smaller than the system size. It seems reasonable to assume that the states will be weakly coupled and consequently will be delocalized over the whole system provided the inequality $\sigma_{\text {eff }} \ll \delta E$ holds. It is remarkable that for $\mu<3 d / 2$, the level spacing $\delta E$ at the top of the band diminishes upon increasing $N$ slower than the effective magnitude of disorder $\sigma_{\text {eff }}$. Therefore, if the coupling between bare states is weak for some finite $N\left(\sigma_{\text {eff }} \ll \delta E\right)$ then it will become even weaker upon increasing $N$. Consequently, one may expect that the state will remain extended in the thermodynamic limit $N \rightarrow \infty$. It is also reasonable to assume that disorder of magnitude larger than the bare bandwidth will localize all the states. From the above arguments we conjectured the existence of an Anderson transition in 1D and 2D systems with diagonal disorder and nonrandom long-range hopping as long as $\mu<3 d / 2$. Below we provide analytical and numerical confirmations of this conjecture.

Concerning the parabolic range of the energy spectrum (close to the bottom of the band), we notice that the level spacing diminishes as $\mathrm{N}^{-2}$ upon increasing the lattice size, i.e., faster than the effective magnitude of disorder $\sigma_{\text {eff }}$. Now, even if $\sigma_{\text {eff }} \ll \delta E$ for a small lattice size and the states are delocalized, the above inequality will be reverted for larger $N$, resulting finally in the localization of those states. The same conclusion holds for both band edges within the nearest-neighbor approximation, where the level spacing is always $\delta E \sim N^{-2}$.

A supersymmetric method for disorder averaging $[14,15]$, combined with a renormalization group (RG) analysis, provide support to the above arguments. In short (the details will be published elsewhere), the sequence of our steps is as follows. As a first step, we consider the one- particle Green's function with the fermionic partition function $Z_{0}$ and the bare action $S_{0}$ defined as

$$
\begin{aligned}
Z_{0} & =\int \prod_{\mathbf{n}} d \psi_{\mathbf{n}} d \bar{\psi}_{\mathbf{n}} e^{-S_{0}}=\operatorname{det}(\mathcal{H}-\mathcal{E} I), \\
S_{0} & =\sum_{\mathbf{n} \mathbf{m}} i \bar{\psi}_{\mathbf{n}}(\mathcal{H}-\mathcal{E} I)_{\mathbf{n m}} \psi_{\mathbf{m}},
\end{aligned}
$$

where $\mathcal{E}=E+i 0^{+}$and $I$ is the identity matrix. Introducing bosonic ghosts $\beta, \bar{\beta}$ and expressing $1 / Z_{0}$ as a path integral [15], we then average the one-particle Green's function using the Gaussian probability distribution of site energies, $P\left(\varepsilon_{\mathbf{n}}\right)=(1 / \pi g)^{1 / 2} \exp \left(-\varepsilon_{\mathbf{n}}^{2} / g\right)$, instead of the box distribution introduced in the beginning. This allows us to perform the integration over site energies explicitly. The effective action

$$
\begin{aligned}
S_{\text {eff }}= & i \sum_{\mathbf{n m}}\left[\bar{\psi}_{\mathbf{n}}\left(J_{\mathbf{n m}}-\mathcal{E} \delta_{\mathbf{n m}}\right) \psi_{\mathbf{m}}+\bar{\beta}_{\mathbf{n}}\left(J_{\mathbf{n m}}-\mathcal{E} \delta_{\mathbf{n m}}\right) \beta_{\mathbf{m}}\right] \\
& +\frac{g}{4} \sum_{\mathbf{n}}\left(\bar{\psi}_{\mathbf{n}} \psi_{\mathbf{n}}+\bar{\beta}_{\mathbf{n}} \beta_{\mathbf{n}}\right)^{2},
\end{aligned}
$$

which appears after averaging, will be the main object of our RG analysis. For doing this, it is convenient to rewrite the action in the $\mathbf{k}$ representation and to regroup the terms as follows: $S_{\text {eff }}=S_{\text {kin }}+S_{\mathcal{E}}+S_{g}$, where

$$
\begin{aligned}
S_{\mathrm{kin}}= & -i J A_{d} \int d^{d} \mathbf{k}|\mathbf{k}|^{\mu-d}[\bar{\psi}(\mathbf{k}) \psi(\mathbf{k})+\bar{\beta}(\mathbf{k}) \beta(\mathbf{k})], \\
S_{\mathcal{E}}= & -i \mathcal{E} \int d^{d} \mathbf{k}[\bar{\psi}(\mathbf{k}) \psi(\mathbf{k})+\bar{\beta}(\mathbf{k}) \beta(\mathbf{k})], \\
S_{g}= & \frac{g}{8 \pi} \int \prod_{i=1}^{4} d^{d} \mathbf{k}_{\mathbf{i}} \delta\left(\mathbf{k}_{\mathbf{1}}+\mathbf{k}_{\mathbf{2}}-\mathbf{k}_{\mathbf{3}}-\mathbf{k}_{\mathbf{4}}\right) \\
& \times\left[\bar{\psi}\left(\mathbf{k}_{\mathbf{1}}\right) \psi\left(\mathbf{k}_{\mathbf{2}}\right)+\bar{\beta}\left(\mathbf{k}_{\mathbf{1}}\right) \beta\left(\mathbf{k}_{\mathbf{2}}\right)\right] \\
& \times\left[\bar{\psi}\left(\mathbf{k}_{\mathbf{3}}\right) \psi\left(\mathbf{k}_{\mathbf{4}}\right)+\bar{\beta}\left(\mathbf{k}_{\mathbf{3}}\right) \beta\left(\mathbf{k}_{\mathbf{4}}\right)\right] .
\end{aligned}
$$

We have absorbed the constant $E_{0}$ into $\mathcal{E}$ in (6b). Note as well that the integration over momenta are restricted to a $d$-dimensional sphere of radius $\Lambda$, i.e., $|\mathbf{k}|<\Lambda$, with $\Lambda$ being an ultraviolet cutoff.

The action (6) is the starting point of our RG analysis, which is inspired by Shankar's approach [16] to fermionic condensed matter systems. The key observation is that the kinetic part of the action, $S_{\text {kin }}$, is invariant under the following scaling transformation of the cutoff $\Lambda$, the momenta $\mathbf{k}$ and the fields:

$$
\begin{aligned}
& \Lambda \rightarrow \Lambda^{\prime}=\Lambda / b, \quad \mathbf{k} \rightarrow \mathbf{k}^{\prime}=b \mathbf{k}, \quad b>1, \\
& \phi\left(\mathbf{k}^{\prime} / b\right)=b^{\mu / 2} \phi\left(\mathbf{k}^{\prime}\right), \quad \phi=\psi, \bar{\psi}, \beta, \bar{\beta} .
\end{aligned}
$$

For generic values of $\mu>d$, Eq. (7a) is a nonstandard scaling law which emerges from the unusual kinetic term (6a). 
Driven by (7), the mass term $S_{\mathcal{E}}$ also transforms into itself with a new coupling constant $\mathcal{E}^{\prime}$ given by $\mathcal{E}^{\prime}=$ $b^{\mu-d} \mathcal{E}$. Thus $\mathcal{E}$ is a relevant perturbation of the free action $S_{\text {kin }}$, as it is always the case of mass terms [16].

The term $S_{g}$ also transforms onto itself under the following RG transformation $g^{\prime}=b^{2 \mu-3 d} g$. This equation implies that $g$ eventually goes to zero upon increasing $b$ provided that $\mu<3 d / 2$. Hence, randomness vanishes in the low energy effective theory for $\mu<3 d / 2$. On the contrary, $g$ runs to stronger coupling whenever $\mu>3 d / 2$.

Finally, the coupling $g$ is marginal at tree level for $\mu=$ $3 d / 2$, and one has to consider the one-loop effects to see its fate. This can be done using the techniques developed in Ref. [16]. Let us present our main results. The RG flows of the constants $g$ and $\mathcal{E}$, up to one loop, are given by

$$
\begin{aligned}
& \frac{d \bar{g}}{d s}=(2 \mu-3 d) \bar{g}+\bar{g}^{2}, \\
& \frac{d \overline{\mathcal{E}}}{d s}=(\mu-d) \overline{\mathcal{E}}-\bar{g},
\end{aligned}
$$

where $s$ is the RG parameter defined as $b=\exp (s)$, and $\bar{g}$ and $\overline{\mathcal{E}}$ are related to $g$ and $\mathcal{E}$ as follows:

$$
g=\frac{\pi}{\Omega_{d}}\left(J A_{d}\right)^{2} \Lambda^{2 \mu-3 d} \bar{g}, \quad \mathcal{E}=\frac{1}{4} J A_{d} \Lambda^{\mu-d} \overline{\mathcal{E}},
$$

with $\Omega_{d}$ being the volume of the $d$-dimensional sphere.

Equation (8a) has an unstable fixed point $\bar{g}_{*}=3 d-$ $2 \mu$ provided that $\mu<3 d / 2$ [see Fig. 1(a)]. Below this point, the coupling $g$ goes to zero asymptotically, while above $g$ grows. For $\mu>3 d / 2$ the critical point disappears and the system always flows to strong coupling [see Fig. 1(b)]. This signals about some changes in the density of states and the different nature of the eigenfunctions when passing from $\mu<3 d / 2$ to $\mu>3 d / 2$. We would like to remark the fact that the critical value $\mu=3 d / 2$ that appeared in the present RG analysis coincides with that found on the basis of our qualitative arguments.

It is usually argued that the average one-particle Green's function does not carry information about the spatial extent of the eigenfunctions. Therefore, we chose the generalized inverse participation ratios (GIPR) as the relevant quantities to characterize localization properties of the states. The standard definition reads $I_{\nu}^{(q)}=$ $\left\langle\sum_{\mathbf{n}}\left|\Psi_{\nu \mathbf{n}}\right|^{2 q}\right\rangle$, where $\Psi_{\nu \mathbf{n}}$ is the probability amplitude of the normalized eigenstate $\nu$ at site $\mathbf{n}$, and the brackets denote disorder averaging. As is well known, the GIPR

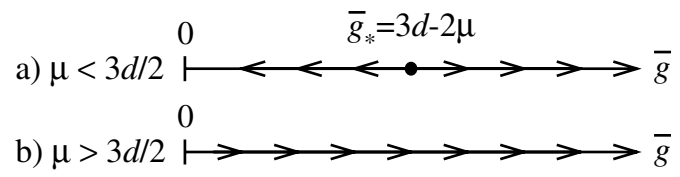

FIG. 1. RG flows for the coupling constant $\bar{g}$, Eqs. (8a) and (9), depending on the value of the exponent $\mu$. scales as $I_{\nu}^{(q)} \sim \mathcal{N}^{-q+1}$ for delocalized states $(q \neq 1)$, while it shows no size scaling for localized ones.

Our main steps in calculating the GIPR are as follows: We estimated the uppermost eigenfunction, $\Psi_{0 \mathbf{n}}$, using perturbation theory with respect to the random term $\sum_{\mathbf{k k}^{\prime}}(\delta \mathcal{H})_{\mathbf{k k}^{\prime}}|\mathbf{k}\rangle\left\langle\mathbf{k}^{\prime}\right|$ in (2a), namely, considering the on-site energies small compared to the hopping parameter $J,\left|\varepsilon_{\mathbf{n}}\right| \ll J$. Then, $\Psi_{0 \mathbf{n}}$ can be written in real space as

$$
\begin{aligned}
\Psi_{0 \mathbf{n}} & =\frac{e^{\phi_{\mathbf{n}}}}{\left(\sum_{\mathbf{n}} e^{2 \phi_{\mathbf{n}}}\right)^{1 / 2}}, \\
\phi_{\mathbf{n}} & \equiv \sum_{\mathbf{m}} S_{\mathbf{n m}} \varepsilon_{\mathbf{m}}, \\
S_{\mathbf{n m}} & \equiv \frac{1}{\mathcal{N}} \sum_{\mathbf{k} \neq \mathbf{0}} \frac{e^{i \mathbf{k}(\mathbf{m}-\mathbf{n})}}{E_{0}-E_{\mathbf{k}}} .
\end{aligned}
$$

In order to compute the GIPR we again made use of the the supersymmetric method for disorder averaging (as described above) with the bare action given by Eq. (5), as well as the replica trick introduced in Ref. [17]. The latter reads

$$
I_{0}^{(q)}=\lim _{r \rightarrow 0} \frac{1}{N^{d}} \sum_{\mathbf{n}, \mathbf{n}_{1}, \ldots, \mathbf{n}_{r-q}}\left\langle e^{2 q \phi_{\mathbf{n}}} \prod_{j=1}^{r-q} e^{2 \phi_{\mathbf{n}_{j}}}\right\rangle .
$$

In doing so, we found that $I_{0}^{(q)} \sim N^{-(q-1) d}$ provided when $d<\mu<3 d / 2$; in other words, the generalized dimension equals the space dimension so that the uppermost state is delocalized, in full agreement with our qualitative picture. In particular, notice that the so-called inverse participation ratio (IPR) scales as $I_{0}^{(2)} \sim \mathcal{N}^{-1}$.

Since the previous analytical study of the GIPR was perturbative, we have also carried out a numerical study of the model to support the validity of our conclusions. We took advantage of the Lanczos method [18] as well as the density matrix renormalization group approach [19], allowing one to calculate some few eigenstates of the Hamiltonian (1) for rather large system size. In Fig. 2 we plotted the IPR $(q=2)$ of the uppermost state as a function of the system size $\mathcal{N}=N^{d}$ for different degrees of disorder $\Delta$. The behavior of the other top states is similar to that which manifests the uppermost state. Observing Fig. 2 we conclude that the uppermost state is delocalized even for a moderately high value of the degree of disorder $(\Delta=8 \mathrm{~J}$ in $1 \mathrm{D}$ systems and $\Delta=40 \mathrm{~J}$ in $2 \mathrm{D}$ system), provided $d<\mu<3 d / 2$. For comparison, the 1D (2D) bandwidth for $\mu=5 / 4(\mu=9 / 4)$ in the absence of disorder is of the order of $10.5 \mathrm{~J}(28 \mathrm{~J})$. However, for a large degree of disorder the IPR remains constant on increasing the system size (see Fig. 2 for $\mu=5 / 4, d=1$, and $\Delta=40 J$ ), indicating that the uppermost eigenstate is localized. Therefore, the top eigenstates undergo the Anderson transition on increasing $\Delta$ whenever $d<\mu<$ $3 d / 2$. It is to be noticed the absence of scaling of the IPR and the subsequent localization for $d=1, \mu=3$, and 


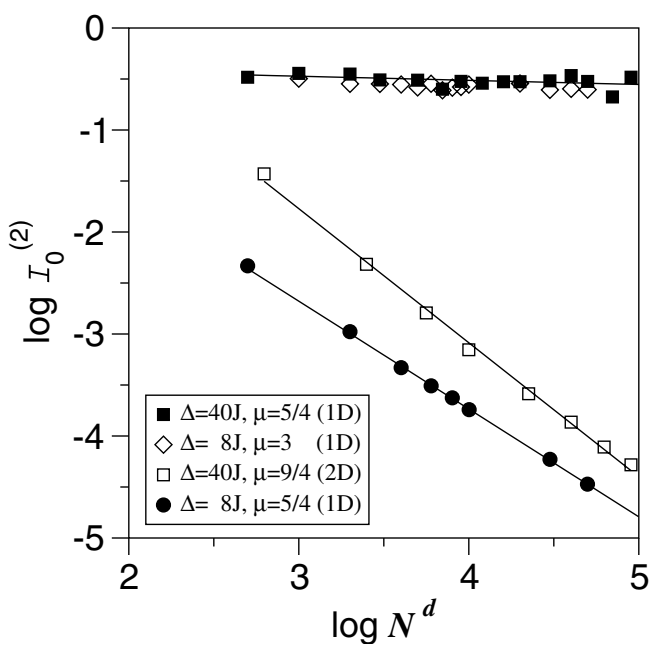

FIG. 2. Scaling of the IPR of the uppermost eigenstate as a function of the number of sites $\mathcal{N}=N^{d}$ for different magnitudes of disorder $\Delta$ in 1D $(d=1)$ and 2D $(d=2)$ lattices.

$\Delta=8 J$. This result is in full correspondence with the analytical analysis stating that no transition is expected in this case.

In summary, we have shown analytically and numerically that a powerlike nonrandom intersite hopping, $J_{\mathbf{n m}}=J /|\mathbf{n}-\mathbf{m}|^{\mu}$, may act towards delocalization of quasiparticle states in low-dimensional systems. In particular, the states of the top of the band may be delocalized at rather high magnitudes of disorder $(\Delta \sim J)$ and undergo a localization-delocalization transition as the magnitude of disorder increases. Scaling arguments provide a clear physical picture of the underlying mechanism responsible for the delocalization of the states, in spite of the low-dimensional $(d \leq 2)$ geometry of the system. The different size scaling of the bare level spacing, $\delta E \sim$ $N^{-\mu+d}$, and the magnitude of disorder seen by a quasiparticle, $\sigma_{\text {eff }} \sim \Delta / N^{d / 2}$, is the feature of the model responsible for this unusual behavior. $\delta E$ decreases slower than $\sigma_{\text {eff }}$ upon increasing the system size as long as $d<$ $\mu<3 d / 2$, resulting in the delocalization of the corresponding quasiparticle states in the thermodynamic limit. We stress that the main finding of our study, namely, the existence of the Anderson transition in a physically relevant model, has been concluded on the basis of three different approaches and the conclusions obtained are self-consistent. Most important, the validity of the scaling analysis is not limited to the present model. Indeed, it is established on solid grounds that the standard, threedimensional Anderson model manifests the localizationdelocalization transition at the band center. Within this model, the bare level spacing at the band center diminishes proportionally to $N^{-1}$, while the magnitude of effective disorder goes down faster, $\sim N^{-3 / 2}$, thus being unable to localize the states at the band center for a moderate disorder. A strong disorder (large compared to the bandwidth) localizes the states, giving rise a localizationdelocalization transition. We can then be confident that this kind of scaling argument may provide physical insight in several localization problems.

V. A. M. acknowledges support from MECyD (Project No. SAB2000-0103). A. R. and F. D-A. were supported by DGI-MCyT (Project No. MAT2000-0734) and CAM (Project No. 07N/0075/2001). G. S. and M. A. M-D. acknowledge support from PGC (Project No. BFM20001320-C02-01).

*On leave from S. I. Vavilov State Optical Institute, SaintPetersburg, Russia.

[1] P.W. Anderson, Phys. Rev. 109, 1492 (1958).

[2] E. Abrahams, P.W. Anderson, D. C. Licciardello, and V. Ramakrishnan, Phys. Rev. Lett. 42, 673 (1979).

[3] P. A. Lee and T.V. Ramakrishnan, Rev. Mod. Phys. 57, 287 (1985).

[4] B. Kramer and A. MacKinnon, Rep. Prog. Phys. 56, 1469 (1993).

[5] D. E. Logan and P. G. Wolynes, Phys. Rev. B 29, 6560 (1984); 31, 2437 (1985); 36, 4135 (1987); J. Chem. Phys. 87, 7199 (1987).

[6] L. S. Levitov, Europhys. Lett. 9, 83 (1989); Ann. Phys. (Leipzig) 8, 697 (1999).

[7] A. D. Mirlin, Y.V. Fyodorov, F.-M. Dittes, J. Quezada, and T. H. Seligman, Phys. Rev. E 54, 3221 (1996).

[8] D. A. Parshin and H. R. Schober, Phys. Rev. B 57, 10232 (1998).

[9] L. I. Deych, A. A. Lisyansky, and B. L. Altshuler, Phys. Rev. Lett. 84, 2678 (2000); Phys. Rev. B 64, 224202 (2001).

[10] A. Nabetani, A. Tamioka, H. Tamaru, and K.. Miyano, J. Chem. Phys. 102, 5109 (1995).

[11] R. Kopelman, M. Shortreed, Z.-Y. Shi, W. Tan, Z. Xu, J. Moore, A. Bar-Haim, and J. Klafter, Phys. Rev. Lett. 78, 1239 (1997).

[12] M. A. Martín-Delgado, J. Rodríguez-Laguna, and G. Sierra, Phys. Rev. B 65, 155116 (2002); cond-mat/ 0012382.

[13] A. Rodríguez, V. A. Malyshev, and F. DomínguezAdame, J. Phys. A 33, L161 (2000).

[14] K. B. Efetov, Adv. Phys. 32, 53 (1983).

[15] S. Guruswamy, A. LeClair, and A.W.W. Ludwig, Nucl. Phys. B583, 475 (2000).

[16] R. Shankar, Rev. Mod. Phys. 66, 129 (1994).

[17] A.W.W. Ludwig, M. P. A. Fisher, R. Shankar, and G. Grinstein, Phys. Rev. B 50, 7526 (1994).

[18] G. H. Golub and C. F. Van Loan, Matrix Computations (The Johns Hopkins University Press, Maryland, 1996).

[19] M. A. Martín-Delgado, G. Sierra, and R. M. Noack, J. Phys. A 32, 6079 (1999). 